\title{
Flocking Motion Control for a System of Nonholonomic Vehicles
}

\author{
Mohammed Abouheaf and Wail Gueaieb \\ School of Information Technology and Engineering \\ University of Ottawa, Ottawa, Ontario, Canada \\ Email: \{Mohammed.Abouheaf, Wail.Gueaieb\}@uottawa.ca
}

\begin{abstract}
The applications of nonholonomic autonomous systems, such as smart cars and wheeled domestic robots, have gained much attention in the scientific and industrial communities. The non-linearities in the kinematics and dynamics of such systems pause many challenges in stabilizing their motion. In this paper, a motion control system with local and team control objectives is introduced for a flock of nonholonomic vehicles, using bounded input-output feedback linearization. The team control objectives include a navigational control system, which is implemented using smooth state feedback control laws, and a synchronization control system, which is implemented using a smooth position dependent adjacency matrix and communication graph structures. The local control objective involves a collision avoidance scheme, which is implemented using an extended Takagi-Sugeno-Kang fuzzy model. The proposed technique is successfully validated in a numerical simulation with 10 differentialdrive mobile robots.
\end{abstract}

\section{INTRODUCTION}

The Autonomous Nonholonomic vehicles have been used in a variety of scientific and industrial applications. These include space exploration, warehouses, computations, communications, and controls [1]-[3]. Designing a controller for the nonholonomic vehicle system is a challenging task due to the nonlinearity of its kinematic and dynamic equations [1]. It was shown that, the nonholonomic system's dynamics cannot be asymptotically stable by applying direct state feedback control laws [4]. The nonholonomic systems can be inputoutput feedback linearized if the output equations are properly selected [5], [6]. Herein, a multi-objective control scheme is introduced to control the motion of a flock of nonholonomic vehicles using cooperative control ideas, smooth potential functions, and fuzzy systems.

The cooperative control problems are divided into synchronization and consensus problems [7], [8]. In the synchronization problem, the agents select their policies to synchronize to the leader's dynamics. In the consensus control problem, the agents reach (or synchronize to) a common value [9]. The cooperative control problem for dynamic multi-agent systems on graphs is solved in [10].

The behavior of large population of systems interacting on communication graph with common goals is known as flocking. Flocking is investigated by researchers from different areas. Flocking applications involve mobile sensor networks, automated delivery of payloads, unmanned air vehicles [11]. The flocking first computer animation was introduced by
Reynolds [12]. Herein, the flocking motion control of multiagent system composed of nonholonomic vehicle systems is studied on graphs, using state feedback control laws, smooth potential functions, and extended TSK-fuzzy models. Where, the control objectives are divided into local and team objectives.

The paper is organized as follows; Section 2, introduces the linear input-output feedback transformation of the nonlinear dynamics of the nonholonomic vehicle system. Section 3, discusses the synchronization and guidance control problems for the flock. Section 4, discusses the separation control problem. Section 5, shows the simulation results for a flock with multi-control objectives.

\section{Problem Formulation; Flock of Nonholonomic VEHICLES}

In the sequel, the motion control of a flock of nonholonomic systems is considered. The dynamic equations of the nonholonomic vehicles satisfying rolling without slipping condition (unicycle type) are considered. Then, the input-output feedback linearization is introduced [1].

\section{A. The Vehicle's Kinematic and Dynamical Equations}

The dynamic equations of each system, shown in Figure 1, are given as follows:

$$
\begin{aligned}
\dot{x}_{1} & =v \cos (\theta), \\
\dot{x}_{2} & =v \sin (\theta), \\
\dot{\theta} & =\omega, \\
\dot{v} & =\frac{1}{l m}\left(\tau_{R}+\tau_{L}\right), \\
\dot{\omega} & =\frac{1}{l J}\left(\tau_{R}-\tau_{L}\right),
\end{aligned}
$$

where $x_{1}, x_{2}$ are the Cartesian coordinates of each agent with center $o, \theta$ is the heading angle, $v, \omega$ are the linear and angular velocities, $m$ is the mass of inertia, $J$ is the moment of inertia, and $\tau_{R}, \tau_{L}$ are the right and left control torques acting on the wheels (control inputs).

The control objectives of the flock would be to let the vehicles track a virtual trajectory, synchronize their speeds to common value, and avoid collisions among each other. The control implementation is challenging, due to the non linearities that exist in the system's dynamics (1). Herein, the input-output feedback linearization proposed in [1], will 


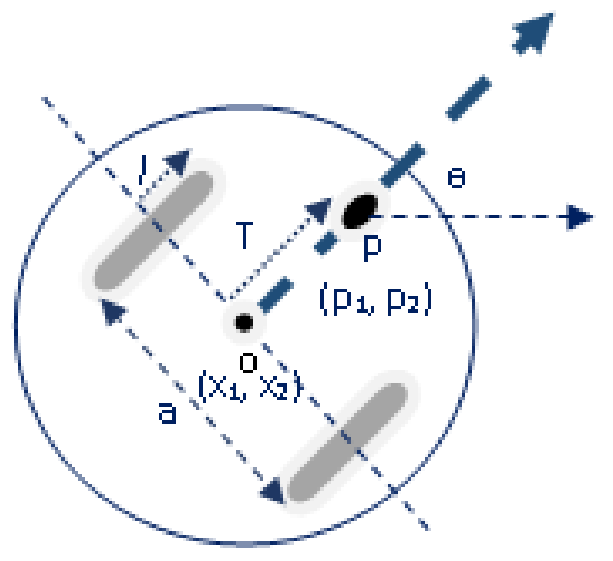

Fig. 1. Nonholonomic Vehicle.

be used to implement the flocking motion control of the nonholonomic systems.

The input-output feedback linearization finds a new reference $p$ related to the center $o$ such that

$$
\left[\begin{array}{l}
p_{1} \\
p_{2}
\end{array}\right]=\left[\begin{array}{c}
x_{1}+T \cos (\theta) \\
x_{2}+T \sin (\theta)
\end{array}\right] .
$$

Considering the following right and left torque expressions [1]

$$
\begin{aligned}
\tau_{R} & =\frac{l m}{2}(\mu-k v)+\frac{l J}{2 a}(\eta-k \omega), \\
\tau_{L} & =\frac{l m}{2}(\mu-k v)-\frac{l J}{2 a}(\eta-k \omega),
\end{aligned}
$$

where $k$ is a positive constant and $\mu$ and $\eta$ are given by [1]

$$
\begin{aligned}
{\left[\begin{array}{l}
\mu \\
\eta
\end{array}\right]=\left[\begin{array}{cc}
\cos (\theta) & \sin (\theta) \\
-\frac{\sin (\theta)}{T} & -\frac{\cos (\theta)}{T}
\end{array}\right] \times } \\
{\left[\begin{array}{c}
u_{1}+v \omega \sin (\theta)+T \omega^{2} \cos (\theta) \\
u_{2}-v \omega \cos (\theta)+T \omega^{2} \sin (\theta)
\end{array}\right] . }
\end{aligned}
$$

Thus, the input-output feedback linear system is given by

$$
\left[\begin{array}{c}
\dot{p}_{1} \\
\dot{p}_{2} \\
\ddot{p}_{1} \\
\ddot{p}_{2}
\end{array}\right]=\left[\begin{array}{cccc}
0 & 0 & 1 & 0 \\
0 & 0 & 0 & 1 \\
0 & 0 & -k & 0 \\
0 & 0 & 0 & -k
\end{array}\right]\left[\begin{array}{c}
p_{1} \\
p_{2} \\
\dot{p}_{1} \\
\dot{p}_{2}
\end{array}\right]+\left[\begin{array}{cc}
0 & 0 \\
0 & 0 \\
1 & 0 \\
0 & 1
\end{array}\right]\left[\begin{array}{l}
u_{1} \\
u_{2}
\end{array}\right]
$$

where $u_{1}$ and $u_{2}$ are the control inputs for each system.

The internal dynamics of the heading angle $\theta$ is given by

$$
\dot{\theta}=-\frac{\dot{p}_{1} \sin \theta}{T}+\frac{\dot{p}_{2} \cos \theta}{T} .
$$

The internal Dynamics are shown to be Lagrange stable in [1].

\section{B. Control Objectives}

The linear input-output feedback transformation (4), motivates the use of the traditional state feedback control laws to control the motion of the nonholonomic systems. The control objectives of the flock are divided into local and team objectives as shown in Figure 2; the local control objective $u^{a}=f^{a}\left(p_{i}, p_{j}\right)$ is to maintain a minimum separation distance between any vehicle $i$ and its neighbor $j, \forall i, j$ (i.e. avoid collision). The team control objectives are twofold; Firstly, each vehicle $i$ is required to synchronize its speed to a common flock speed $u^{s}=f^{s}\left(p_{i}, p_{j}\right)$ and secondly, each vehicle is required to track a virtual trajectory $u^{t r}=f^{t r}\left(p_{i}, \dot{p}_{i}, p_{r}, \dot{p}_{r}\right)$. Thus, the overall objectives can be combined together for each control input $u$ of the system (4) such that

$$
u=f^{t r}\left(p_{i}, \dot{p}_{i}, p_{r}, \dot{p}_{r}\right)+f^{a}\left(p_{i}, p_{j}\right)+f^{s}\left(p_{i}, p_{j}\right) .
$$

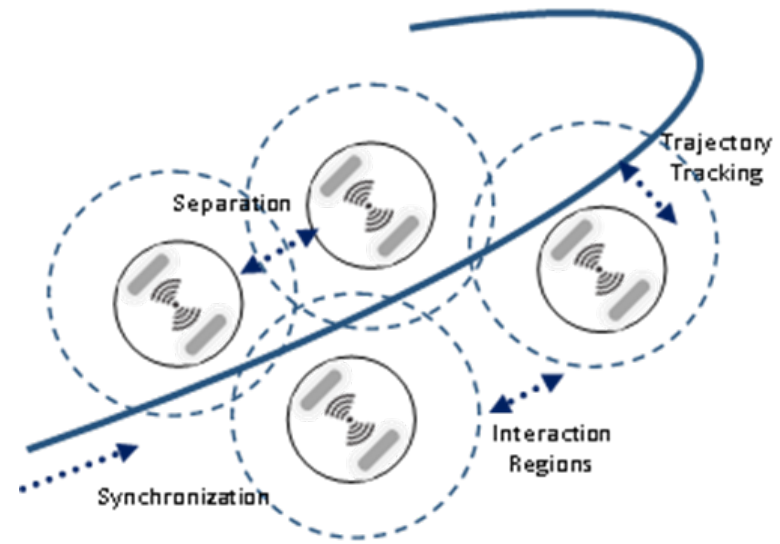

Fig. 2. Control Objectives for a Flock of Nonholonomic Vehicles.

\section{The Synchronization AND GuidAnCE CONTROL PROBLEMS}

In this section, the synchronization of the vehicles is achieved using communication graph structures and adjacency matrices which are built on smooth adjacency elements [11]. The connection weights available to each system are changing continuously depending on the vehicle's interaction radius (this interaction region defines the proximity and the communication structure among the vehicles). Consequently, the graph Laplacian will be a position dependent matrix. Finally, simple state feedback control laws can be used to achieve the required navigation and guidance between the vehicles to the virtual trajectory.

\section{A. Graph Structure}

The graph $G$ is defined by a set of vertices $\nu$ and a set of edges $\varepsilon$. The connectivity (adjacency) matrix $A=\left[a_{i j}\right]=$ $f\left(p_{i}, p_{j}\right),\{i, j \in \nu, j \neq i\}$ of the graph is a varying dependent position matrix with nonzero elements satisfying $a_{i j} \neq 0 \Leftrightarrow$ $(j, i) \in \varepsilon$. The smooth adjacency elements limit the adjacency matrix's coefficients (matrix entries) to values between 0 and 1 [11]. The set of neighbors of each vehicle $i$ can be defined as $N_{i}=\{j \in \nu:(i, j) \in \varepsilon\}$. 


\section{B. Graph Laplacian: Synchronization Results}

The graph properties enable the flock to reach consensus on the velocity provided that, the graph is strongly connected (strongly connected graph implies existence of a spanning tree). The graph Laplacian $L$ describes the team objectives of the nonholonomic systems. For a graph $G$ of order $N$ with adjacency matrix $A$ and in-degree matrix $D$ ( $D$ is a diagonal matrix with diagonal entries $d_{i}=\sum_{j=1}^{N} a_{i j}$ ), the graph Laplacian $(L)$ is given by $L=D-A$.

Remark 1: The second eigenvalue $\lambda_{2}(L)$ associated with the Fiedler Eigenvector, determines how fast the vehicles could reach a common value. In addition, motion forces like, the collision avoidance forces and the navigational forces act together and influence the synchronization speed.

\section{Smooth Adjacency Matrix}

A scalar pump function $\rho_{h}\left(p_{i j}\right)$ [11] is used to construct the smooth adjacency matrix $A$ and it is given by

$$
\rho_{h}\left(p_{i j}\right)= \begin{cases}1 & , p_{i j} \in[0, h) \\ \frac{1}{2}\left[1+\cos \left(\pi \frac{p_{i j}-h}{1-h}\right)\right] & , p_{i j} \in[h, 1), \\ 0 & , \text { otherwise }\end{cases}
$$

where $p_{i j}$ is the distance between any two adjacent systems $i$ and $j$.

Remark 2: The pump function (7) not only decides the connectivity weights of the graph (which affects the speed of convergence) but also, "implicitly" reflects into the separation control efforts. This is because, a decision about the weight is taken based on the non-allowed or the conflict area between the vehicles.

The elements of the proximity net adjacency matrix $A\left(p_{i j}\right)$ are given by

$$
a_{i j}\left(p_{i j}\right)=\rho_{h}\left(\left\|p_{j}-p_{i}\right\|_{\delta} /\|r\|_{\delta}\right) \in[0,1), \quad j \neq i .
$$

Remark 3: The $\|\cdot\|_{\sigma}$ or $\sigma$-norm is a mapping $R^{m} \rightarrow R \geq$ 0 , which is differentiable everywhere unlike the regular norms $\|\cdot\|$ and it is given by

$$
\left\|p_{i j}\right\|_{\sigma}=\frac{1}{\varepsilon}\left[\sqrt{1+\varepsilon\left\|p_{i j}\right\|^{2}}-1\right]
$$

where $\varepsilon$ is a positive real number.

\section{Synchronization Control Laws}

In terms of the adjacency matrix (8) and the respective smooth adjacency elements (7). The synchronization control law is given by

$$
u^{s}=-L\left(p_{i j}\right) P,
$$

where $P$ is a vector of the position elements.

Remark 4: The control objectives (6), "implicitly" ensure a dynamically changing and strongly connected graph, if the repulsive forces are acting on a vehicle, the navigational or the guidance forces will send the vehicle back to the flock.

\section{E. Navigational Control Laws}

The vehicles are required to track a pre-planned or virtual trajectory. The virtual trajectory is given by

$$
p^{t r}=\left[\begin{array}{l}
p_{1 t r} \\
p_{2 t r}
\end{array}\right]=\left[\begin{array}{c}
5 \sin (0.5 T) \\
5 \cos (0.5 T)
\end{array}\right] .
$$

Thus, the virtual dynamical model can be given by

$$
\begin{aligned}
& \dot{x}_{1 t r}=x_{2 t r} \\
& \dot{x}_{2 t r}=f\left(x_{1 t r}, x_{2 t r}\right)
\end{aligned},
$$

where $x_{1 t r}$ is the trajectory for one of the Cartesian coordinates.

The linearized system (4), enables the use of direct state feedback control laws to implement the navigational control objective such that

$$
u_{1(2)}^{t r}=-z_{1}\left(p_{1(2)}-p_{1(2) t r}\right)-z_{2}\left(\dot{p}_{1(2)}-\dot{p}_{1(2) t r}\right),
$$

where $z_{1}$ and $z_{2}$ are positive feedback gains.

\section{The Separation Control Problem}

In this section, the separation control law is implemented using the extended Takagi-Sugeno-Kang (TSK) Fuzzy systems.

\section{A. Extended TSK Fuzzy Model}

The extended TSK fuzzy system is an affine model, where the consequences are affine in the antecedent parts [13]. Thus, the system with $m$ inputs and single output can be implemented using $n$ rules such that

$$
\begin{aligned}
& \text { Rule } i: \text { if } \delta_{1} \text { is } \psi_{1 i}, . . \text {, and } \delta_{m} \text { is } \psi_{m i} \\
& \text { Then } \zeta_{i}=\phi_{i} \delta,
\end{aligned}
$$

where $\delta$ is an input vector for rule $i$; where $\delta=\left[\delta_{1}, \delta_{2}, . ., \delta_{n}\right]$, $\psi_{j i}$ is the fuzzy set of input $j$ and rule $i, \phi_{i}$ is output coefficient of rule $i$, and the function $\zeta_{i}$ is the consequence of rule $i$.

The extended Gaussian membership functions are used for the input fuzzy sets such that

$$
\psi(\rho)=\exp \left[-\left|\frac{\rho-\alpha}{\beta}\right|^{c}\right],
$$

where $c \geq 0, \alpha \in R, \beta \succ 0$.

This function represents a family of curves [14]. Thus, numerous membership functions can be obtained by changing the value of the constant $c$ (for example; triangle, trapezoid, and Gaussian-shaped membership functions) [14]. The TSK Fuzzy model using this type of membership functions, is denoted by extended TSK Fuzzy system. The traditional TSK Fuzzy model is considered a sub category of the extended TSK fuzzy systems.

The firing strength of rule $i$ is determined by the product of all activated membership functions $\prod_{j=1}^{m} \psi_{j i}\left(\delta_{j}\right)$. The output of the extended TSK fuzzy model $\zeta$ interpolates the decisions given by the different rules. The output is considered as a normalized weighted average of the consequences of the $n$ rules such that

$$
\zeta=\sum_{i=1}^{n}\left(\prod_{j=1}^{m} \psi_{j i}\left(\delta_{j}\right)\right) \zeta_{i}(\delta) .
$$




\section{B. The Separation Control Problem}

The separation control law is implemented using the output of the TSK fuzzy model. Thus, a smooth potential function is considered [16]. The agents are required to maintain a minimum space $d$, which is the global minimum of that potential function. Moreover, the vehicle has a limited interaction communication range.

Remark 5: The communication graph appears, in the antecedent parts and consequently, the consequence parts of the fuzzy rules (The smooth potential function depends on the communication structure available to each vehicle system).

Each rule $q$ can be designed such that

$$
\begin{aligned}
& \text { Rule } q \\
& \text { if: }\left\|p_{1 j}-p_{1 i}\right\|_{\sigma}-\|d\|_{\sigma} \text { is } \psi_{q} \\
& \text { Then: } \\
& \zeta_{q}=\phi_{q} \rho_{h}\left(\left\|p_{1 j}-p_{1 i}\right\|_{\delta} /\|r\|_{\delta}\right) \frac{p_{1 j}-p_{1 i}}{\sqrt{1+\varepsilon\left\|p_{1 j}-p_{1 i}\right\|^{2}}} \text {. }
\end{aligned}
$$

Thus, the separation control law for each Cartesian coordinate is given by

$$
\begin{aligned}
& u_{1(2)}^{a}=\sum_{q} \psi_{q} \frac{\left(\left\|p_{1(2) j}-p_{1(2) i}\right\|_{\sigma}-\|d\|_{\sigma}\right) \phi_{q}}{\phi_{q}} \times \\
& \rho_{h}\left(\left\|p_{1(2) j}-p_{1(2) i}\right\|_{\delta} /\|r\|_{\delta}\right) \frac{p_{1(2) j}-p_{1(2) i}}{\sqrt{1+\varepsilon\left\|p_{1(2) j}-p_{1(2) i}\right\|^{2}}} .
\end{aligned}
$$

\section{Simulation Results}

The simulation is performed to test the effectiveness of the combined control structures when applied simultaneously. The multi-objective control scheme is tested for a flock of ten nonholonomic systems. The control objectives are summarized as follows:

- The vehicles are required to track a virtual trajectory.

- The vehicles are required to avoid collision.

- The vehicles are required to synchronize to a common speed.

The flock parameters are chosen such that:

$k=8.23, d=0.5, r=0.75, h=0.2, z_{1}=6.5, z_{2}=6.5$.

Eight rules are considered with the following fuzzy set parameters:

$\psi=\left[\begin{array}{lllllllll}L N & N & S N & Z & S P & P & L P & P P\end{array}\right]$,

$\phi=\left[\begin{array}{llllllll}-150 & -100 & -70 & 0 & 2 & 3 & 4\end{array}\right]$.

The fuzzy model structure is inspired by [15], [16] .

Three simulation cases are considered to test the robustness of the control scheme.

\section{A. Case I: Tracking Circular Trajectory}

In this simulation case, the vehicles are required to track a curricular trajectory. The parameters of the membership functions of the antecedent parts are given as follows:

$\alpha=\left[\begin{array}{llllllll}-1.5 & -1 & -0.5 & 0 & 1 & 2 & 4 & 5\end{array}\right], \beta=1.5$.

The simulation is performed and the control signals are applied simultaneously. Figure 3 (Phase Plan Plot) shows the tracking and guidance properties of the flock. The vehicles track the desired circular trajectory and at the same time they keep a minimum distance to avoid collision. Figure 4 shows the group speed of the flock. This figure highlights the asymptotic synchronization properties of the flock.

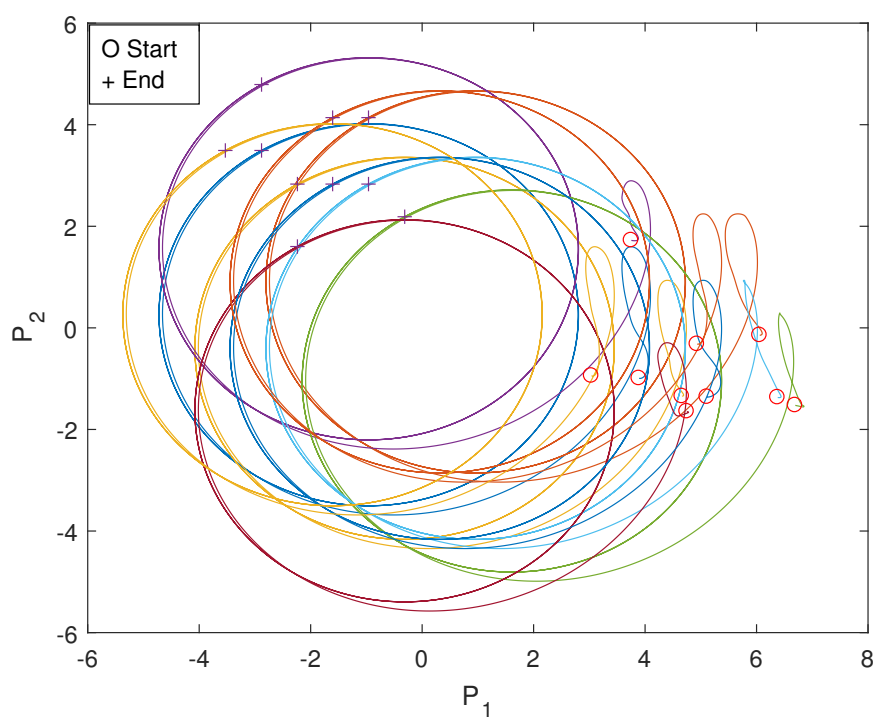

Fig. 3. Phase Plan Plot.
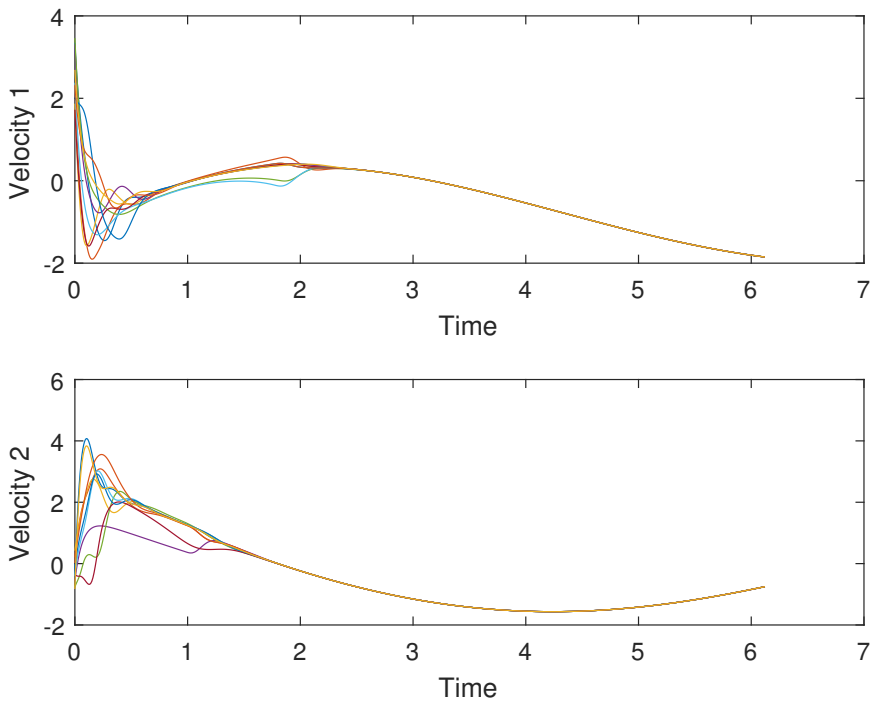

Fig. 4. Synchronization.

\section{B. Case II: Tracking The Center of the Flock}

In this simulation case, the vehicles are required to track the mean of the position vectors of the flock. Thus, the tracking control strategy depends on the means of the position vectors of the vehicles. The same gain parameters from the previous simulation case are used. The value $d$ is chosen such that $(d=1)$.

The vehicles started from scattered positions as shown in Figure 5. The simulation shows that, the tracking control 
strategy will bring the vehicles from the scattered positions until they become in the effective communication range for each other. This enables the other control strategies to have more influence to control the synchronization and separation of the vehicles. The synchronization is achieved as shown in Figure 6.

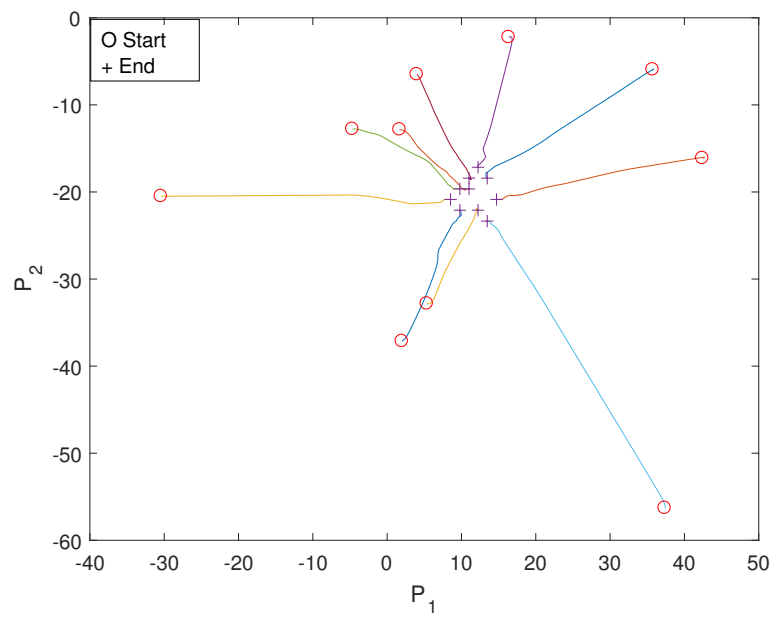

Fig. 5. Phase Plan Plot.
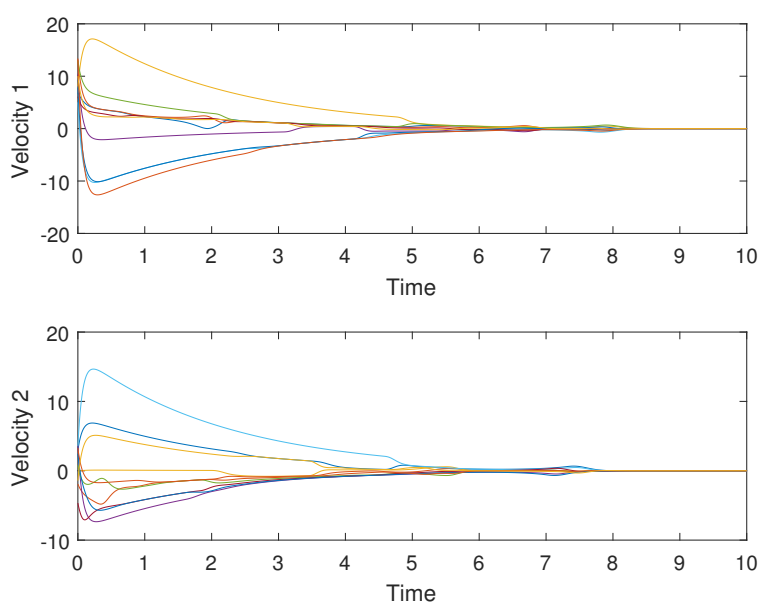

Fig. 6. Synchronization.

\section{Case III: Mamdany Fuzzy System}

In this simulation case, a Mamdany Fuzzy System is considered to implement the separation control objective. The vehicles are required to track the curricular trajectory. The system uses the following membership function

$$
\Psi(\rho)= \begin{cases}0 & , \rho \leq i_{1} \\ \frac{\rho-i_{1}}{i_{2}-i_{1}} & , i_{1} \leq \rho \leq i_{2} \\ 1 & , i_{2} \leq \rho \leq i_{3} \\ \frac{i_{4}-\rho}{i_{4}-i_{3}} & , i_{3} \leq \rho \leq i_{4} \\ 0 & , i_{4} \leq \rho\end{cases}
$$

The membership functions of the consequence parts have the following parameters $i_{1}=\left[\begin{array}{lllllll}-3 & -2 & - & 1 & 0 & 1 & 1.5\end{array}-1-\right.$

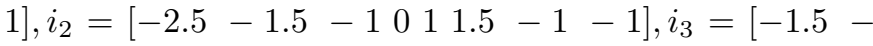

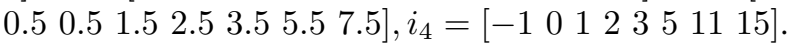

Thus, the separation control law for each Cartesian coordinate is given by

$$
\begin{aligned}
& u_{1(2) M}^{a}=\operatorname{sign}\left(p_{1(2) j}-p_{1(2) i}\right) \times \\
& \sum_{q} \psi_{q} \frac{\left(\left\|p_{1(2) j}-p_{1(2) i}\right\|_{\sigma}-\|d\|_{\sigma}\right) \phi_{q}}{\phi_{q}} \times \\
& \rho_{h}\left(\left\|p_{1(2) j}-p_{1(2) i}\right\|_{\delta} /\|r\|_{\delta}\right) .
\end{aligned}
$$

Figure 7 shows the phase plan plot of the flock. It is shown that, the vehicles are following the circular trajectory. The vehicles also maintain a minimum separation distance. Figure 8 shows the speed properties of the flock.

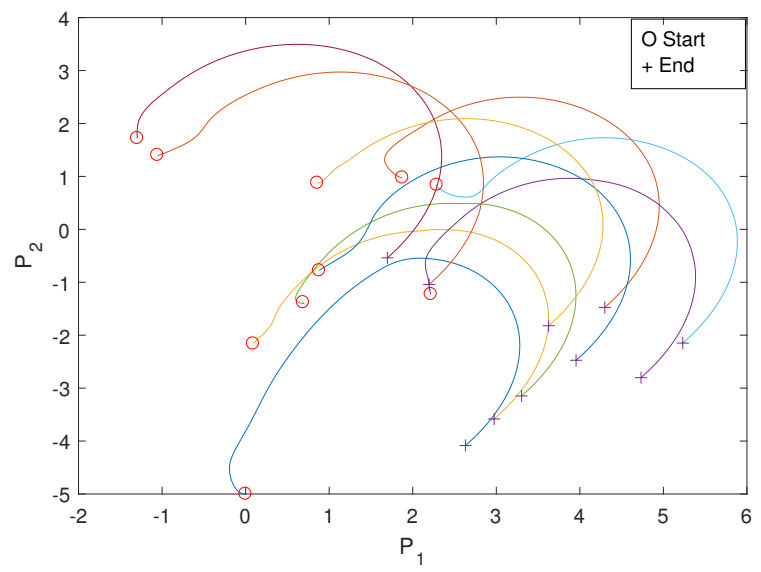

Fig. 7. Phase Plan Plot.

The introduced simulation cases emphasize the robustness of the introduced multi-objective control structure. This system works for different fuzzy systems and for different tracking objectives.

\section{CONCLUSION}

A multi-objective control scheme is introduced to control the motion of a flock of nonholonomic systems. This scheme is implemented using smooth potential functions and state feedback control laws. The Input-output feedback linearization of the nonholonomic kinematic constraints facilitated the use 

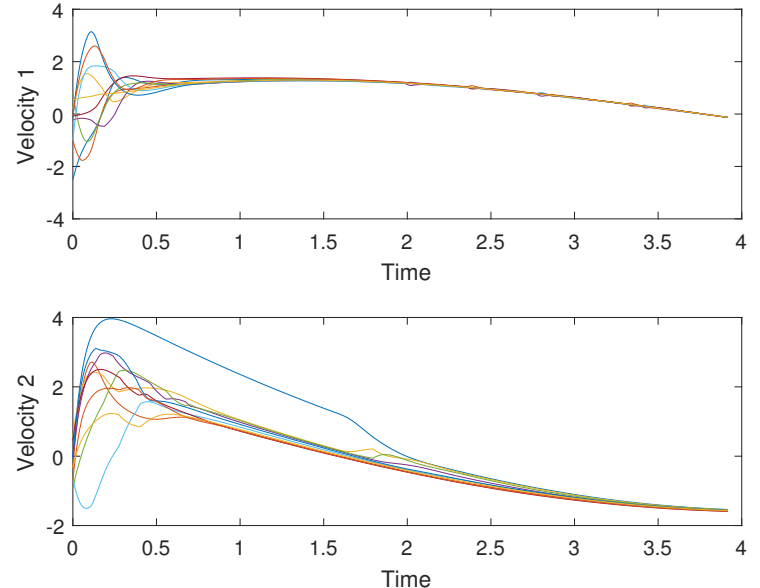

Fig. 8. Synchronization.

of the linear state feedback control laws to achieve the guidance and navigation control goals. The synchronization among the vehicles is achieved using smooth adjacency matrices, where the communication graph structure uses smooth position dependent matrices. The separation control goal is achieved using extended TSK Fuzzy model.

\section{REFERENCES}

[1] E. Rodriguez-Seda, C. Tang, M. Spong, D. Stipanovic "Trajectory tracking with collision avoidance for nonholonomic vehicles with acceleration constraints and limited sensing," The International Journal of Robotics Research, 33(12), pp. 1569-1592, 2014.

[2] R. Bogue 'Robots for space exploration,'Industrial Robot, 39(4), pp. 323 328, 2012.

[3] P. Wurman, R. DAndrea, and M. Mountz, "Coordinating hundreds of cooperative, autonomous vehicles in warehouses," AI Magazine, 29(1), pp. 9-20, 2008.

[4] R. Brockett, Asymptotic stability and feedback stabilization. In: Brockett RW, Millman RS and Sussmann HJ (eds) Differential Geometric Control Theory. Boston, MA: Birkhauser, pp. 181-191, 1983.

[5] N. Sarkar, X. Yun, and V. Kumar, "Control of mechanical sys-tems with rolling constraints: Application to dynamic control of mobile robots," International Journal of Robotics Research, 13(1) pp. 55-69, 1994.

[6] Y. Yamamoto and X. Yun, "Coordinating locomotion and manipulation of a mobile manipulator," IEEE Transactions on Automation and Control, 39(6), pp. 1326-1332, 1994.

[7] R. Beard and V. Stepanyan, Synchronization of information in distributed multiple vehicle coordination control, Proc. of the IEEE Conf on Dec and Con, Maui, HI, pp., 2029-2034, 2003.

[8] J. Tsitsiklis. Problems in Decentralized Decision Making and Computation. Ph.D. dissertation. Dept. Elect. Eng. and Comput. Sci., MIT, Cambridge, MA, 1984.

[9] Z. Li, Z. Duan, G. Chen, and L. Huang, Consensus of multi-agent systems and synchronization of complex networks: A unified viewpoint, IEEE Trans Circ \& Syst, vol. 57, no. 1, pp. 213-224, 2010.

[10] M. Abouheaf, F. Lewis, M. Mahmoud, and D. Mikulski, Discretetime dynamic graphical games: model-free reinforcement learning solution, Cont.Thr. \& Techn., vol.13, no.1, pp. 55-60, 2015.

[11] R. O. Sabry, Flocking for Multi-Agent Dynamic Systems:Algorithms and Theory, IEEE Trans. on Auto. Con., vol. 51, No. 3, March 2006.

[12] C. W. Reynolds. Flocks, herds, and school: a distributed behavioural model. Computer Graphics CACM SIGGRAPH' 87 , 21(4):25 34, 1987.

[13] H. O. Wang, J. Li, D. Niemann, and K. Tanaka, T-S model with linear rule consequence and PDC controller: A universal framework for nonlinear control systems, in Proc. IEEE Int. Conf. Fuzzy Syst., Arlington,pp. 549$554,2000$.
[14] W. J. Fang, L. W. Hau, Based on Extended T-S Fuzzy Model of Selfadaptive Disturbed PSO Algorithm, Second International Conference on Information and Computing Science, pp.150-153, 2009.

[15] D. Gu, H. Hu, Using Fuzzy Logic to Design Separation Function in Flocking Algorithms, IEEE Trans on Fuzzy Syst., vol. 16, No. 4, pp. 826838, August 2008.

[16] H. Yu, T. Thang, Center Guided Flocking Motion of Multi-agent Using Fuzzy Logic, Proceedings - 2009 International Conference on Computational Intelligence and Software Engineering, CiSE 2009. 